\title{
Verification of Logic Programs with Delay Declarations
}

\author{
Krzysztof R. Apt ${ }^{1,2}$ and Ingrid Luitjes ${ }^{2}$ \\ 1 CWI, P.O. Box 94079, 1090 GB Amsterdam, The Netherlands \\ 2 Department of Mathematics and Computer Science \\ University of Amsterdam, Plantage Muidergracht 24 \\ 1018 TV Amsterdam, The Netherlands
}

\begin{abstract}
Logic programs augmented with delay declarations form a higly expressive programming language in which dynamic networks of processes that communicate asynchronously by means of multiparty channels can be easily created. In this paper we study correctness these programs. In particular, we propose proof methods allowing us to deal with occur check freedom, absence of deadlock, absence of errors in presence of arithmetic relations, and termination. These methods turn out to be simple modifications of the corresponding methods dealing with Prolog programs. This allows us to derive correct delay declarations by analyzing Prolog programs. Finally, we point out difficulties concerning proofs of termination.
\end{abstract}

Note. The research of the first author was partly supported by the ESPRIT Basic Research Action 6810 (Compulog 2).

\section{Introduction}

In Kowalski [Kow79] the slogan "Algorithm = Logic + Control" was coined. This paper suggested logic programming as a formalism for a systematic development of algorithms. The idea was to endow a logic program with a control mechanism to obtain an executable program. Prolog is a realization of this idea where the control consists of the leftmost selection rule combined with the depth-first search in the resulting search tree.

But this idea of a control is in many cases overly restrictive. As an extreme example consider a theorem prover written in Prolog. A proof rule

$$
\frac{A_{1}, \ldots, A_{n}}{B}
$$

naturally translates into a Prolog clause

$$
\operatorname{prove}(B) \leftarrow \operatorname{prove}\left(\mathbb{A}_{1}\right), \ldots, \operatorname{prove}\left(\Lambda_{n}\right)
$$

according to which the premises $A_{1}, \ldots, A_{n}$ have to be proved in the abovementioned order. In contrast, in the underlying logic the order in which the premises are to be proved is usually arbitrary.

In general, however, some order between the actions of a logic program is necessary. As an example consider the QUICKSORT program: 


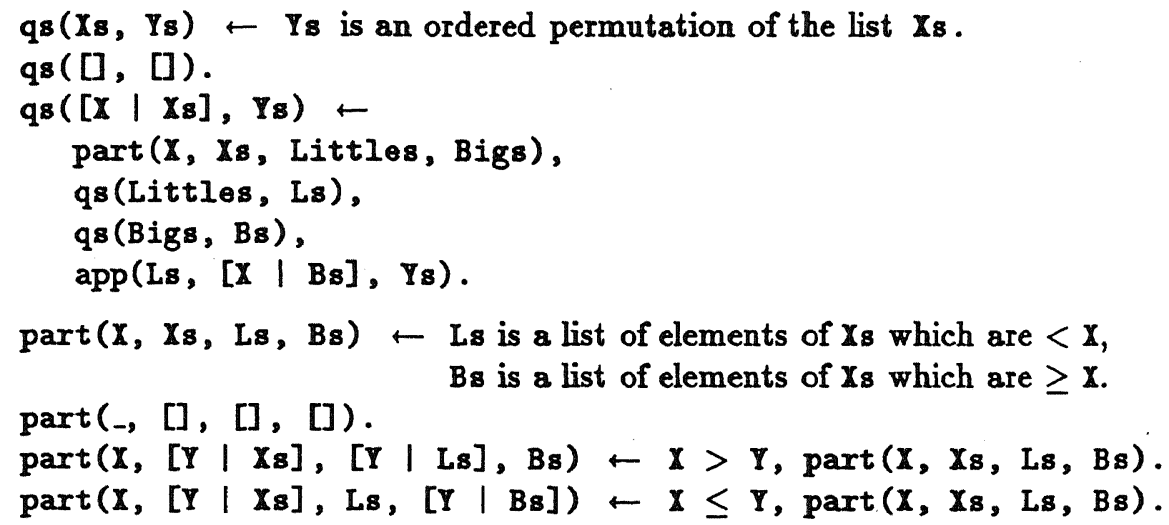

augmented by the following APPEND program:

$\operatorname{app}\left(X_{s}, Y_{s}, Z_{s}\right) \leftarrow Z_{s}$ is the result of concatenating the lists $X_{s}$ and $Y_{s}$. $\operatorname{app}\left([], Y_{s}, Y_{s}\right)$.

$\operatorname{app}\left(\left[\begin{array}{l|l}X & X_{8}\end{array}\right], Y_{8},\left[X \mid Z_{8}\right]\right) \leftarrow \operatorname{app}\left(X_{8}, Y_{8}, Z_{8}\right)$.

It is easy to see that starting with a query qs ( $\mathrm{s}, \mathrm{Ys})$, where $s$ is a list of integers, QUICKSORT diverges when the rightmost atom is repeatedly selected. Also, a run time error results if the arithmetic atoms $X>Y$ and $X \leq Y$ are selected "too early". To prevent this type of undesired behaviour some coordination between the actions of the program is necessary.

For this purpose in Naish [Nai82] delay declarations were proposed. The idea is to replace the Prolog selection rule by a more flexible selection mechanism according to which atoms are delayed until they become "sufficiently" instantiated. This is achieved by adding to the program so-called delay declarations. Then at each stage of the execution of a logic program only atoms satisfying the delay declarations can be selected. In presence of trivial delay declarations any atom can be selected and a nondeterministic logic program without any control declaration is then obtained.

More recently, the delay declarations were studied in Lüttringhaus-Kappel [LK93] and also incorporated in various versions of Prolog, notably Sicstus Prolog, and in Gödel, the programming language proposed by Hill and Lloyd [HL94]. Actually, in all these references a more restricted selection rule is employed, according to which the leftmost non-delayed atom is selected. This selection rule allows us to model within the logic programming the coroutine mechanism and lazy evaluation. In contrast, the selection rules here studied. and originally considered in Naish [Nai88], allow us to model parallel executions.

Returning to the above QUICKSORT program we note that the coordination between the program actions has to do with the need to produce the appropriate "inputs" before executing the "calls" which use them. The requirement to compute these inputs can be expressed by means of the following delay declarations, where the last two declarations are meant to ensure that the arithmetic relations are called with the right inputs: 


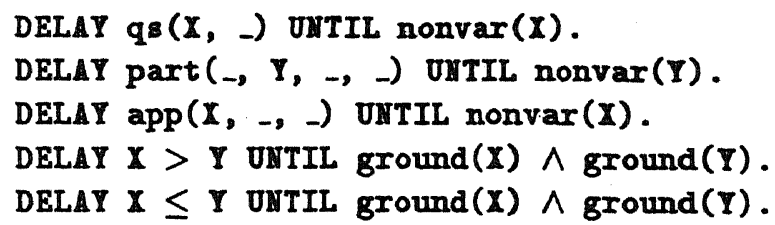

The behaviour of the resulting program is highly non-trivial, since during its executions dynamic networks of asynchronously communicating processes are created.

Delay declarations form a powerful control mechanism. In general, we can identify three natural uses of them:

- to enforce termination,

- to prevent absence of errors in presence of arithmetic operations,

- to impose a synchronization between various actions of the program; this makes it possible to model parallel executions.

In this paper we illustrate each of these uses of delay declarations and provide formal means of justifying them. More specifically, we study here various correctness aspects of logic programs in presence of delay declarations, visibly occur check freedom, absence of deadlock, absence of errors in presence of arithmetic operations, and termination. In each case we propose a simple method which can be readily applied to several well-known programs.

These results imply that for the query $q_{8}\left(\mathrm{~s}, \mathrm{Y}_{\mathrm{s}}\right)$, where $\mathrm{s}$ is a list of integers, QUICKSORT augmented by the above delay declarations is occur-check free and deadlock free. Moreover, no errors due to the presence of arithmetic operations arise and under some additional assumptions all derivations terminate.

Interestingly, the suggested proof methods turn out to be simple modifications of the corresponding methods dealing with Prolog programs. So the transition from Prolog to programs with delay declarations is quite natural, even though the latter ones permit more execution sequences and more complex "intermediate situations". This observation is further substantiated by showing how "correct" delay declarations can be derived by analyzing Prolog programs so that the given Prolog program can be excecuted in a more flexible way.

\section{Preliminaries}

In what follows we use the standard notation of Lloyd [Llo87] and Apt [Apt90], though we work here with queries, that is sequences of atoms, instead of goals, that is constructs of the form $\leftarrow Q$, where $Q$ is a query. In particular, given a syntactic construct $E$ (so for example, a term, an atom or a set of term equations) we denote by $\operatorname{Var}(E)$ the set of the variables appearing in $E$. Recall that an mgu $\theta$ of a set of term equations $E$ is called relevant if $\operatorname{Var}(\theta) \subseteq \operatorname{Var}(E)$.

The following lemma (see e.g. Apt and Pellegrini [AP94]) will be needed in Section 6 . 
Lemma 1 (Iteration). Let $E_{1}, E_{2}$ be two sets of term equations. Suppose that $\theta_{1}$ is a relevant mgu of $E_{1}$ and $\theta_{2}$ is a relevant mgu of $E_{2} \theta_{1}$. Then $\theta_{1} \theta_{2}$ is a relevant $m g u$ of $E_{1} \cup E_{2}$. Moreover, if $E_{1} \cup E_{2}$ is unifiable then a relevant mgu $\theta_{1}$ of $E_{1}$ exists, and for any mgu $\theta_{1}$ of $E_{1}$ a relevant mgu $\theta_{2}$ of $E_{2} \theta_{1}$ exists, as well.

We now define the syntax of delay declarations. We loosely follow here Hill and Lloyd [HL94]. First, we define inductively a set of conditions:

- true is a condition,

- given a variable $X$, ground $(X)$ and nonvar $(X)$ are conditions,

- if $c_{1}$ and $c_{2}$ are conditions, then $c_{1} \wedge c_{2}$ and $c_{1} \vee c_{2}$ are conditions.

Next, we define inductively when an instance of a condition holds:

- true holds,

- ground( $\mathrm{s}$ ) holds if $\mathrm{s}$ is a ground term,

- nonvar(s) holds if $s$ is a non-variable term,

$-c_{1} \wedge c_{2}$ holds if $c_{1}$ and $c_{2}$ hold,

$-c_{1} \vee c_{2}$ holds if $c_{1}$ or $c_{2}$ holds.

Call an atom a $p$-atom if its relation symbol is $p$. A delay declaration associated for a relation symbol $p$ has the form DELAY 1 UNTIL COND, where $A$ is a $p$-atom and COND is a condition. From now on we consider logic programs augmented by the delay declarations, one for each of its relation symbols. In the presentation below we drop the delay declarations of the form DELAY 1 UITIL true.

The following simple definition explains the use of delay declarations.

\section{Definition 2.}

- We say that an atom B satisfies a delay declaration DELAY $A$ UNTIL COND if for some substitution $\theta$ both $B=\Lambda \theta$ and $\operatorname{covin} \theta$ hold.

In particular, if $x_{1}, \ldots, x_{n}$ are different variables, then $p\left(s_{1}, \ldots, s_{n}\right)$ satisfies a delay declaration DELAY $p\left(X_{1}, \ldots, X_{n}\right)$ UNTIL COND if COND $\left\{x_{1} / s_{1}, \ldots, x_{n} / s_{n}\right\}$ holds.

- An SLD-derivation respects the delay declarations if all atoms selected in it satisfy their delay declarations.

Intuitively, in presence of delay declarations only atoms which satisfy their delay declarations can be selected. So in presence of delay declarations we allow only those selection rules which generate SLD-derivations respecting the delay declarations. Note that in presence of delay declarations a query can be generated in which no atom can be selected because none of them satisfies its delay declaration. We view such a fragment of an SLD-derivation as a finite SLD-derivation.

In what follows we shall study correctness of logic programs augmented with the delay declarations. To show the usefulness of the obtained results and to 
see their limitations, we shall analyze in this paper three example programs: QUICKSORT from the introduction, and the following two.

The program IH_ORDER constructs the list of all nodes of a binary tree by means of an in-order traversal:

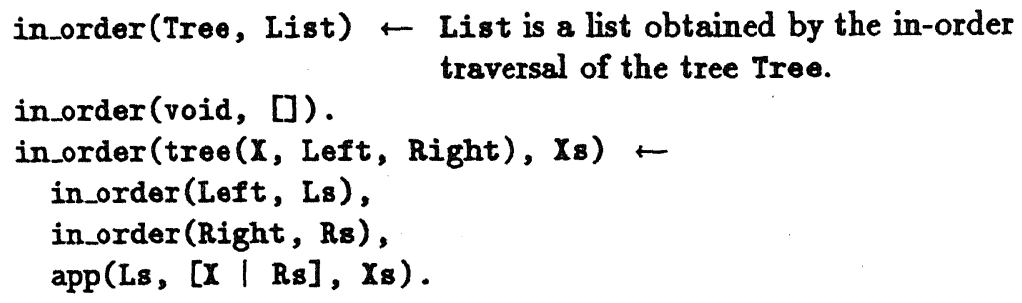

augmented by the APPEND program.

together with the following delay declarations:

DELAY in_order $(X,-)$ UNTIL nonvar $(X)$.

DELAY app (X, , - ) UNTIL nonvar(X).

Finally, the program SEQUENCE (see Coelho and Cotta [CC88, page 193]) solves the following problem: arrange three 1's, three 2's, ..., three 9's in sequence so that for all $i \in[1,9]$ there are exactly $i$ numbers between successive occurrences of $i$.

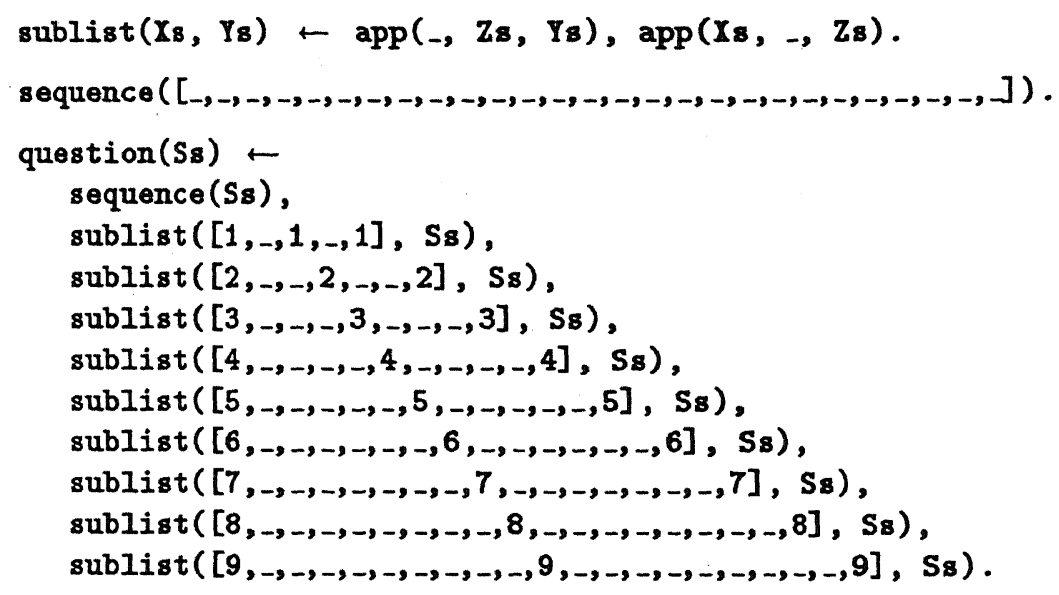

augmented by the APPEND program.

together with the following the delay declaration:

DELAY app (_, , Z) UNTIL nonvar(Z).

\section{Occur-check Freedom}

In most Prolog implementations for the efficiency reasons so-called occur-check is omitted from the unification algorithm. It is well-known that this omission 
can lead to incorrect results. The resulting difficulties are usually called the occur-check problem. They have motivated a study of conditions under which the occur-check can be safely omitted. In this section we study this problem for logic programs augmented with delay declarations. To this end we recall the relevant definitions. We follow here Apt and Pellegrini [AP94] though we adapt its framework to arbitrary SLD-derivations. The following notion is due to Deransart, Ferrand and Téguia [DFT91].

Definition 3. A system of term equations $E$ is called not subject to occur-check (NSTO in short) if no execution of the Martelli-Montanari unification algorithm (see Martelli and Montanari [MM82]) started with $E$ ends with a system that includes an equation of the form $x=t$, where $x$ is a variable and $t$ a term different from $x$, but in which $x$ occurs.

We can now introduce the crucial notion of this section.

\section{Definition 4.}

- Consider an SLD-derivation $\xi$. Let $A$ be an atom selected in $\xi$ and $H$ the head of the input clause selected to resolve $A$ in $\xi$. Suppose that $A$ and $H$ have the same relation symbol. Then we say that the system $A=H$ is considered in $\xi$.

- An SLD-derivation is called occur-check free if all the systems of equations considered in it are NSTO.

Recall that in presence of delay declarations selection of an atom implies that it satisfies its delay declaration.

In what follows we identify some syntactic conditions that allow us to draw conclusions about the occur-check freedom. To this end we use modes. Informally, modes indicate how the arguments of a relation should be used.

Definition5. Consider an $n$-ary relation symbol $p$. By a mode for $p$ we mean a function $m_{p}$ from $\{1, \ldots, n\}$ to the set $\{+,-\}$. If $m_{p}(i)=$ 't', we call $i$ an input position of $p$ and if $m_{p}(i)=$ '-', we call $i$ an output position of $p$ (both w.r.t. $\left.m_{p}\right)$. By a moding we mean a collection of modes, each for a different relation symbol.

We write $m_{p}$ in a more suggestive form $p\left(m_{p}(1), \ldots, m_{p}(n)\right)$. For example, member $(-,+)$ denotes a binary relation member with the first position moded as output and the second position moded as input.

The definition of moding assumes one mode per relation in a program. Multiple modes may be obtained by simply renaming the relations. In this paper we adopt the following

Assumption Every considered relation has a fixed mode associated with it.

This assumption will allow us to talk about input positions and output positions of an atom. 


\section{Definition 6.}

- A family of terms is called linear if every variable occurs at most once in it.

- An atom is called input (resp. output) linear if the family of terms occurring in its input (resp. output) positions is linear.

- An atom is called input-output disjoint if the family of terms occurring in its input positions has no variable in common with the family of terms occurring in its output positions.

In the sequel we shall use the following lemma of Apt and Pellegrini [AP94].

Lemma 7. Consider two atoms $A$ and $H$ with the same relation symbol. Suppose that

- they have no variable in common,

- one of them is input-output disjoint,

- one of them is input linear and the other is output linear.

Then $A=H$ is NSTO.

The first result is an immediate consequence of Lemma 7. The idea is that when the head of every clause of $P$ is output linear, it suffices to delay all the atoms until their input positions become ground. To this end we introduce the following notion that relates the delay declarations to modes.

Definition 8. We say that the delay declarations imply the moding if every atom which satisfies its delay declaration is ground in its input positions.

Theorem $\theta$ (Occur-check Freedom 1). Suppose that

- the head of every clause of $P$ is output linear,

- the delay declarations imply the moding.

Then all $S L D$-derivations of $P \cup\{Q\}$ which respect the delay declarations are occur-check free.

Proof. By Lemma 7.

This result allows us to deal only with the delay "until ground" declarations. So for example, we cannot draw yet at this stage any conclusions concerning the QUICKSORT program from the introduction. To deal with other forms of delay declarations we use the following notion introduced in Chadha and Plaisted [CP94] and further studied in Apt and Pellegrini [AP94].

To simplify the notation, when writing an atom as $p(u, v)$, we now assume that $\mathbf{u}$ is a sequence of terms filling in the input positions of $p$ and that $v$ is $a$ sequence of terms filling in the output positions of $p$. 


\section{Definition 10.}

- A query $p_{1}\left(\mathbf{s}_{1}, t_{1}\right), \ldots, p_{n}\left(s_{n}, t_{n}\right)$ is called nicely moded if $t_{1}, \ldots, t_{n}$ is a linear family of terms and for $i \in[1, n]$

$$
\operatorname{Var}\left(\mathbf{s}_{\mathbf{i}}\right) \cap\left(\bigcup_{j=i}^{n} \operatorname{Var}\left(\mathbf{t}_{\mathbf{j}}\right)\right)=0 .
$$

- A clause

$$
p_{0}\left(\mathbf{s}_{0}, t_{0}\right) \leftarrow p_{1}\left(\mathbf{s}_{1}, t_{1}\right), \ldots, p_{n}\left(s_{n}, t_{n}\right)
$$

is called nicely moded if $\leftarrow p_{1}\left(s_{1}, t_{1}\right), \ldots, p_{n}\left(s_{n}, t_{n}\right)$ is nicely moded and

$$
\operatorname{Var}\left(\mathbf{s}_{0}\right) \cap\left(\bigcup_{j=1}^{n} \operatorname{Var}\left(\mathbf{t}_{\mathbf{j}}\right)\right)=\emptyset \text {. }
$$

In particular, every unit clause is nicely-moded.

- A program is called nicely moded if every clause of it is.

Intuitively, the concept of being nicely moded prevents a "speculative binding" of the variables which occur in output positions - these variables are required to be "fresh". Note that a query with only one atom is nicely moded iff it is output linear and input-output disjoint.

The following lemma is crucial. It shows persistence of the notion of nice modedness in presence of a natural assumption.

Lemma 11 (Nice modedness). Every SLD-resolvent of a nicely moded query and a nicely moded clause with an input-linear head, that is variable-disjoint with it, is nicely moded.

Proof. The proof is quite long and can be found in Luitjes [Lui94]. It is similar to the proof of an analogous lemma, 5.3, in Apt and Pellegrini [AP94, pages 719-724].

Corollary 12 (Nice modedness). Let $P$ and $Q$ be nicely moded. Suppose that the head of every clause of $P$ is input linear. Then all queries in all SLDderivations of $P \cup\{Q\}$ are nicely moded.

This corollary brings us to the following conclusion.

Theorem 13 (Occur-check Freedom 2). Let $P$ and $Q$ be nicely moded. Suppose that

- the head of every clause of $P$ is input linear.

Then all $S L D$-derivations of $P \cup\{Q\}$ are occur-check free.

Proof. By the Nice modedness Corollary 12 all queries in all SLD-derivations of $P \cup\{Q\}$ are nicely moded. But every atom of a nicely moded query is inputoutput disjoint and output linear. So the claim follows by Lemma 7. 
This result shows that for nicely moded programs and queries occur-check freedom can be ensured inpendendently of the selection rule, so without taking into account the delay declarations. In Chadha and Plaisted [CP94] and Apt and Pellegrini [AP94] it was shown that the QUICKSORT program with the moding qs $(+,-)$, partition $(+,+,-,-)$, app $(+,+,-),+>+,+\leq+$ satisfies the condition of the above theorem, so this result applies to any query of the form qs (s, Ys), where $\mathbf{s}$ is a list of integers.

To apply the above result to the IH_ORDER program consider the following moding: in_order $(+,-)$, app $(+,+,-)$. It is straightforward to check that then III_ORDER is nicely moded and the head of every clause of IN_ORDER is input linear.. So by the Occur-check Freedom 2 Theorem 13 we conclude that for any term $t$ and $a$ variable $Y_{s}$ that does not occur in $t$, all SLD-derivations of II _ORDER $\cup\{$ in_order $(t$, Ys $)\}$ are occur-check free.

Finally, to deal with the SEQUENCE program take the following moding: sublist $(-,+)$, sequence $(+)$, question $(+)$, app $(-,-,+)$. Thanks to the use of anonymous variables it is easy to check that SEQUENCE is then indeed nicely moded and that the heads of all clauses are input linear. So by the Occur-check Freedom 2 Theorem 13 all SLD-derivations of SEqUENCE $\cup\{$ question(Ss)\} are occur-check free.

\section{Absence of Deadlock}

In presence of delay declarations a query can be generated in which no atom can be selected, because none of them satisfies its delay declaration. Then the computation cannot proceed. Such a situation is obviously undesirable. We call it a deadlock and study here means to avoid it. Let us begin with a formal definition.

Definition 14. An SLD-derivation flounders if it contains a query no atom of which satisfies its delay declaration. We say that $P \cup\{Q\}$ deadlocks if an SLDderivation of $P \cup\{Q\}$ which respects the delay declarations flounders.

We now propose syntactic conditions which allow us to prove absence of deadlock. The main tool is the notion of a well-moded program and query. Let us recall the definition (see e.g. Dembinski and Maluszynski [DM85] and Drabent
[Dra87]).

\subsection{Well-moded Queries and Programs}

Definition 15.

- A query $p_{1}\left(s_{1}, t_{1}\right), \ldots, p_{n}\left(s_{n}, t_{n}\right)$ is called well-moded if for $i \in[1, n]$

$$
\operatorname{Var}\left(\mathbf{s}_{\mathbf{i}}\right) \subseteq \bigcup_{j=1}^{i-1} \operatorname{Var}\left(\mathbf{t}_{\mathbf{j}}\right)
$$


- A clause

$$
p_{0}\left(t_{0}, \mathbf{s}_{\mathbf{n}+1}\right) \leftarrow p_{1}\left(\mathbf{s}_{1}, \mathbf{t}_{1}\right), \ldots, p_{n}\left(\mathbf{s}_{\mathbf{n}}, \mathbf{t}_{\mathbf{n}}\right)
$$

is called well-moded if for $i \in[1, n+1]$

$$
\operatorname{Var}\left(\mathbf{s}_{\mathbf{i}}\right) \subseteq \bigcup_{j=0}^{i-1} \operatorname{Var}\left(\mathrm{t}_{\mathbf{j}}\right)
$$

- A program is called well-moded if every clause of it is.

The following lemma shows the persistence of the notion of well-modedness. It strengthens the version given in Apt and Pellegrini [AP94] to arbitrary SLDresolvents.

Lemma 16 (Well-modedness). Every SLD-resolvent of a well-moded query and a well-moded clause, that is variable-disjoint with it, is well-moded.

Proof. An SLD-resolvent of a query and a clause is obtained by means of the following three operations:

- instantiation of a query,

- instantiation of a clause,

- replacement of an atom, say $H$, of a query by the body of a clause whose head is $H$.

So we only need to prove the following two claims.

Claim 1 An instance of a well-moded query (resp. clause) is well-moded.

Proof. It suffices to note that for any sequences of terms $s, t_{1}, \ldots, t_{n}$ and a substitution $\theta, \operatorname{Var}(\mathbf{s}) \subseteq \bigcup_{j=1}^{n} \operatorname{Var}\left(\mathrm{t}_{j}\right)$ implies $\operatorname{Var}(\mathbf{s} \theta) \subseteq \bigcup_{j=1}^{n} \operatorname{Var}\left(\mathrm{t}_{j} \theta\right)$.

Claim 2 Suppose that $\mathbf{A}, H, \mathbf{C}$ is a well-moded query and $H \leftarrow \mathbf{B}$ is a wellmoded clause. Then $\mathbf{A}, \mathbf{B}, \mathbf{C}$ is a well-moded query.

Proof. Let

$$
\begin{aligned}
& \mathbf{A}:=p_{1}\left(\mathbf{s}_{1}, \mathrm{t}_{1}\right), \ldots, p_{k}\left(\mathbf{s}_{k}, \mathbf{t}_{k}\right), \\
& H:=p(\mathbf{s}, \mathbf{t}) \\
& \mathbf{B}:=p_{k+1}\left(\mathbf{s}_{k+1}, \mathbf{t}_{k+1}\right), \ldots, p_{k+l}\left(\mathbf{s}_{k+l}, \mathbf{t}_{k+l}\right), \\
& \mathbf{C}:=p_{k+l+1}\left(\mathbf{s}_{k+l+1}, \mathbf{t}_{k+l+1}\right), \ldots, p_{n}\left(\mathbf{s}_{k+l+m}, \mathbf{t}_{k+l+m}\right) .
\end{aligned}
$$

Fix now $i \in[1, k+l+m]$. We need to prove that $\operatorname{Var}\left(\mathbf{s}_{i}\right) \subseteq \bigcup_{j=1}^{i-1} \operatorname{Var}\left(\mathbf{t}_{j}\right)$. Case 1 i $\in[1, k]$.

Note that $\mathbf{A}$ is well-moded, since $\mathbf{A}, H, \mathbf{C}$ is well-moded. Hence the claim follows. Case $2 i \in[k+1, k+l]$.

$H \leftarrow \mathbf{B}$ is well-moded, so $\operatorname{Var}\left(\mathbf{s}_{\mathbf{i}}\right) \subseteq \operatorname{Var}(\mathbf{s}) \cup \bigcup_{j=k+1}^{i-1} \operatorname{Var}\left(\mathbf{t}_{j}\right)$. Moreover, $\mathbf{A}, H, \mathbf{C}$ is well-moded, so $\operatorname{Var}(\mathbf{s}) \subseteq \bigcup_{j=1}^{k} \operatorname{Var}\left(\mathrm{t}_{j}\right)$. This implies the claim. 
Case $3 i \in[k+l+1, k+l+m]$.

$\mathbf{A}, H, \mathbf{C}$ is well-moded, so $\operatorname{Var}\left(\mathrm{s}_{i}\right) \subseteq \bigcup_{j=1}^{k} \operatorname{Var}\left(\mathrm{t}_{j}\right) \cup \operatorname{Var}(\mathrm{t}) \cup \bigcup_{j=k+l+1}^{i-1} \operatorname{Var}\left(\mathrm{t}_{j}\right)$ and $\operatorname{Var}(\mathbf{s}) \subseteq \bigcup_{j=1}^{k} \operatorname{Var}\left(\mathrm{t}_{j}\right)$. Moreover, $H \leftarrow \mathbf{B}$ is well-moded, so

$$
\operatorname{Var}(\mathrm{t}) \subseteq \operatorname{Var}(\mathrm{s}) \cup \bigcup_{j=k+1}^{k+l} \operatorname{Var}\left(\mathrm{t}_{j}\right) .
$$

This implies the claim.

Corollary 17 (Well-modedness). Let $P$ and $Q$ be well-moded. Then all queries in all $S L D$-derivations of $P \cup\{Q\}$ are well-moded.

The following definition provides a link between the delay declarations and moding.

Definition 18. We say that the delay declarations are implied by the moding if every atom which is ground in its input positions satisfies its delay declaration.

We can now state and prove the desired result.

Theorem 19 (Absence of Deadlock 1). Let $P$ and $Q$ be well-moded. Suppose that the delay declarations are implied by the moding. Then $P \cup\{Q\}$ does not deadlock.

Proof. By the Well-modedness Corollary 17 all queries in all SLD-derivations of $P \cup\{Q\}$ are well-moded. But the first atom of a well-moded query is ground in its input positions. Hence, by the assumption, in every SLD-derivation of $P \cup\{Q\}$ the first atom of every query satisfies its delay declaration. Consequently, no SLD-derivation of $P \cup\{Q\}$ flounders.

Intuitively, the above result states that under the abovementioned conditions, at every stage of a computation the first atom can always be selected.

The above result can be applied to the QUICKSORT program. Indeed, with the moding considered in Section 3 QUICKSORT is easily seen to be well-moded, the query $q \mathrm{~s}(\mathrm{~s}, \mathrm{Y} / \mathrm{s})$, where $\mathrm{s}$ is a list of integers, is well-moded and the delay declarations considered in the introduction are clearly implied by this moding. In fact, the same reasoning applies when the original delay declarations are strengthened by replacing everywhere "nonvar" by "ground".

The Absence of Deadlock 1 Theorem 19 can also be used for the IH_ORDER program. Indeed, in the moding considered in the previous section IH_ORDER is clearly well-moded. So the above theorem is applicable to any query of the form in_order $\left(t, y_{s}\right)$, where $t$ is a ground term. However, no conclusion can be drawn if $t$ is not ground. Below we shall see how to draw such stronger conclusions. 
Finally, the above theorem cannot be applied to the program SEQUENCE. Indeed, it is easy to see that no moding exists for which both SEQUENCE and the query question(Ss) are well-moded. So the above theorem cannot be applied to the SEQUENCE program no matter what delay declarations are used.

\subsection{Well-typed Queries and Programs}

To overcome these difficulties we generalize the above approach by using the notion of a type. The presentation below of well-typed queries and programs is taken from Apt and Etalle [AE93]. We begin by adopting the following general definition.

Definition 20. A type is an non-empty set of terms closed under substitution.

We now fix a specific set of types, denoted by Types, which includes:

$U$ - the set of all terms,

List - the set of lists,

Gae - the set of of all ground arithmetic expressions (gae's, in short),

ListGae - the set of lists of gae's.

Tree - the set of binary trees, defined inductively as follows: void is a tree and if $s, t$ are trees, then for any term $u, \operatorname{tre\theta }(u, s, t)$ is a tree.

Of course, the use of the type List assumes the existence of the empty list [] and the list constructor [.I.] in the language, etc.

We call a construct of the form $s: S$, where $s$ is a term and $S$ is a type, a typed term. Given a sequence $8: \mathbf{S}=s_{1}: S_{1}, \ldots, s_{n}: S_{n}$ of typed terms, we write $s \in S$ if for $i \in[1, n]$ we have $s_{i} \in S_{i}$. Further, we abbreviate the sequence $s_{1} \theta, \ldots, s_{n} \theta$ to $s \theta$. Finally, we write

$$
\vDash \mathbf{s}: \mathbf{S} \Rightarrow \mathbf{t}: \mathbf{T},
$$

if for all substitutions $\theta, \mathbf{s} \theta \in \mathbf{S}$ implies $\mathbf{t} \theta \in \mathbf{T}$.

Next, we define types for relations.

Definition 21. By a type for an $n$-ary relation symbolp we mean a function $t_{p}$ from $[1, n]$ to the set Types. If $t_{p}(i)=T$, we call $T$ the type associated with the position $i$ of $p$.

In the remainder of this paper we consider a combination of modes and types and adopt the following

Assumption Every considered relation has a fixed mode and a fixed type associated with it.

This assumption will allow us to talk about types of input positions and of output positions of an atom. An $n$-ary relation $p$ with a mode $m_{p}$ and type $t_{p}$ will be denoted by

$$
p\left(m_{p}(1): t_{p}(1), \ldots, m_{p}(n): t_{p}(n)\right)
$$


For example, member $(-: U,+:$ List $)$ denotes a binary relation member with the first position moded as output and typed as $U$, and the second position moded as input and typed as List.

To simplify the notation, when writing an atom as $p(\mathbf{u}: \mathbf{S}, \mathbf{v}: \mathbf{T})$ we now assume that $\mathbf{u}: \mathbf{S}$ is a sequence of typed terms filling in the input positions of $p$ and $v: T$ is a sequence of typed terms filling in the output positions of $p$. We call a construct of the form $p(\mathbf{u}: \mathbf{S}, \mathbf{v}: \mathbf{T})$ a typed atom and a sequence of typed atoms a typed query. We say that a typed atom $p\left(s_{1}: S_{1}, \ldots, s_{n}: S_{n}\right)$ is correctly typed in position $i$ if $s_{i} \in S_{i}$ and use an analogous terminology for typed queries.

The following notion is due to Bronsard, Lakshman and Reddy [BLR92].

\section{Definition 22.}

- A query

$$
p_{1}\left(\mathbf{i}_{1}: \mathbf{I}_{\mathbf{1}}, \mathbf{o}_{\mathbf{1}}: \mathbf{O}_{\mathbf{1}}\right), \ldots, p_{\mathbf{n}}\left(\mathbf{i}_{\mathbf{n}}: \mathbf{I}_{\mathbf{n}}, \mathbf{o}_{\mathbf{n}}: \mathbf{O}_{\mathbf{n}}\right)
$$

is called well-typed if for $j \in[1, n]$

$$
\vDash o_{1}: o_{1}, \ldots, o_{j-1}: o_{j-1} \Rightarrow i_{j}: I_{j} \text {. }
$$

- A clause

$$
p_{0}\left(\mathbf{o}_{\mathbf{0}}: \mathbf{O}_{\mathbf{0}}, \mathbf{i}_{\mathbf{n}+\mathbf{1}}: \mathbf{I}_{\mathbf{n}+\mathbf{1}}\right) \leftarrow p_{1}\left(\mathbf{i}_{\mathbf{1}}: \mathbf{I}_{\mathbf{1}}, \mathbf{o}_{\mathbf{1}}: \mathbf{O}_{\mathbf{1}}\right), \ldots, p_{\mathbf{n}}\left(\mathbf{i}_{\mathbf{n}}: \mathbf{I}_{\mathbf{n}}, \mathbf{o}_{\mathbf{n}}: \mathbf{O}_{\mathbf{n}}\right)
$$

is called well-typed if for $j \in[1, n+1]$

$$
\vDash \mathbf{o}_{\mathbf{0}}: \mathbf{O}_{\mathbf{0}}, \ldots, \mathbf{o}_{\mathbf{j}-\mathbf{1}}: \mathbf{O}_{\mathbf{j}-\mathbf{1}} \Rightarrow \mathbf{i}_{\mathbf{j}}: \mathbf{I}_{\mathbf{j}}
$$

- A program is called well-typed if every clause of it is.

Note that a query with only one atom is well-typed iff this atom is correctly typed in its input positions. The following lemma shows persistence of the notion of well-typedness. It strenghtens a result mentioned in Bronsard, Lakshman and Reddy [BLR92] to arbitrary SLD-resolvents.

Lemma 23 (Well-typedness). Every SLD-resolvent of a well-typed query and a well-typed clause, that is variable-disjoint with it, is well-typed.

Proof. The proof is analogous to that of the Well-modedness Lemma 16 and is omitted.

Corollary 24 (Well-typedness). Let $P$ and $Q$ be well-typed. Then all queries in all SLD-derivations of $P \cup\{Q\}$ are well-typed.

Finally, we link the delay declarations with types.

Definition 25. We say that the delay declarations are implied by the typing if every atom which is correctly typed in its input positions satisfies its delay declaration.

We can now state and prove the desired result. 
Theorem 26 (Absence of Deadlock 2). Let $P$ and $Q$ be well-typed. Suppose that the delay declarations are implied by the typing. Then $P \cup\{Q\}$ does not deadlock.

Proof. By the Well-typedness Corollary 24 all queries in all SLD-derivations of $P \cup\{Q\}$ are well-typed. But the first atom of a well-typed query is correctly typed in its input positions. Hence, by the assumption, in every SLD-derivation of $P \cup\{Q\}$ the first atom of every query satisfies its delay declaration. Consequently, no SLD-derivation of $P \cup\{Q\}$ flounders.

Let us see how to apply this theorem to the IN_ORDER program. Consider the following typing:

in_order (+: Tree, - : List), app(+ : List, + : List, + : List).

We leave to the reader the task of checking that IN_ORDER is then well-typed and that the delay declarations are implied by the typing. We conclude that for any term $t$ which is a tree, IN_ORDER $U\{$ in_order $(t, Y s)\}$ does not deadlock, which strengthens the conclusion drawn by means of the Absence of Deadlock 1 Theorem 19.

Finally, note that this theorem can also be applied to the SEQUENCE program. Indeed, consider the following typing:

question(- : List),

sequence $(-:$ List $)$,

sublist (+ : List, $+:$ List),

app(- : List, - : List, + : List).

Again, it is easy to see that SEQUENCE and the query question(Ss) are then well-typed and that the delay declaration is implied by the typing. It is worthwhile to note that if we change in this declaration "nonvar" to "ground", then SEQUENCE $\cup\{$ question(Ss)\} does deadlock.

\section{Absence of Errors}

One of the natural uses of the delay declarations is to prevent run time errors in presence of arithmetic relations. This is for example the idea behind the delay declarations

DELAY $X>Y$ UNTIL ground(X) $\wedge$ ground(Y).

DELAY $X \leq Y$ ONTIL ground $(X) \wedge$ ground $(Y)$.

used in the introduction which ensure that both relations are called only with ground arguments. Now, to prove absence of errors a stronger property is needed, namely that the arguments of these relations are ground arithmetic expressions. However, the syntax of the delay declarations does not allow us to express this stronger information.

The aim of this section is to provide means to deduce this stronger property and thus to prove absence of errors in presence of arithmetic relations. To this 
end we shall use the notions of a well-typed query and a well-typed program introduced in the previous section.

The following simple observation provides us with some means to prove that if an atom is ground in its input positions, then it is correctly typed in its input positions.

Lemma 27. Suppose that $\mathbf{A}, B, \mathbf{C}$ is a well-typed query such that

- $B$ is ground in its input positions,

- for some substitution $\theta, \mathbf{A} \theta$ is correctly typed in its output positions.

Then $B$ is correctly typed in its input positions.

Proof. Let $\mathbf{A}=p_{1}\left(\mathbf{i}_{1}: \mathbf{I}_{1}, o_{1}: \mathbf{O}_{1}\right), \ldots, p_{n}\left(\mathbf{i}_{\mathbf{n}}: \mathbf{I}_{\mathbf{n}}, \mathbf{O}_{\mathbf{n}}: \mathbf{O}_{\mathbf{n}}\right)$ and

$B=p_{n+1}\left(\mathbf{i}_{\mathbf{n}+1}: \mathbf{I}_{\mathbf{n}+1}, \mathbf{o}_{\mathbf{n}+1}: \mathbf{O}_{\mathbf{n}+1}\right)$. By the definition of well-typedness

$$
\vDash o_{1}: o_{1}, \ldots, o_{n}: o_{n} \Rightarrow i_{n+1}: I_{n+1} \text {. }
$$

But by the assumption $o_{i} \theta \in O_{i}$ for $i \in[1, n]$, so $i_{n+1} \theta \in I_{n+1}$ which implies the claim since by assumption $i_{n+1} \theta=i_{n+1}$.

We need to apply this lemma at every stage of the SLD-derivations. To prove the second assumption we shall use the following notion introduced in Apt and Etalle [AE93].

\section{Definition 28.}

- A query $p_{1}\left(s_{1}, t_{1}\right), \ldots, p_{n}\left(s_{n}, t_{n}\right)$ is called simply moded if $t_{1}, \ldots, t_{n}$ is a linear family of variables and for $i \in[1, n]$

$$
\operatorname{Var}\left(\mathbf{s}_{\mathbf{i}}\right) \cap\left(\bigcup_{j=i}^{n} \operatorname{Var}\left(\mathbf{t}_{\mathbf{j}}\right)\right)=\emptyset
$$

- A clause

$$
p_{0}\left(\mathbf{s}_{0}, t_{0}\right) \leftarrow p_{1}\left(\mathbf{s}_{1}, t_{1}\right), \ldots, p_{n}\left(\mathbf{s}_{\mathbf{n}}, t_{\mathbf{n}}\right)
$$

is called simply moded if $p_{1}\left(\mathbf{s}_{1}, \mathbf{t}_{1}\right), \ldots, p_{n}\left(\mathbf{s}_{\mathbf{n}}, \mathbf{t}_{\mathbf{n}}\right)$ is simply moded and

$$
\operatorname{Var}\left(\mathbf{s}_{\mathbf{0}}\right) \cap\left(\bigcup_{j=1}^{n} \operatorname{Var}\left(\mathbf{t}_{\mathbf{j}}\right)\right)=0 \text {. }
$$

In particular, every unit clause is simply moded.

- A program is called simply moded if every clause of it is.

So simple modedness is a special case of nice modedness. The sole difference lies in the new assumption that each output position of a query or of a body of a clause is filled by a variable. The following lemma clarifies our interest in the notion of simple modedness. 
Lemma 28. Let $\mathbb{A}$ be a typed query which is simply moded. Then for some substitution $\theta, \mathbb{A} \theta$ is correctly typed in its output positions.

Proof. By the definition the output positions of $\mathbb{A}$ are filled by different variables. Choose for each variable occurring in an output position of $\mathbb{A}$ a term from the type associated with this position. So obtained bindings form the desidered substitution.

The next step is to prove persistence of the notion of simple modedness. In Apt and Etalle [AE93] this property is established for the SLD-derivations w.r.t. the leftmost selection rule. But the generalization to arbitrary SLD-derivations does not work, as the following example shows. Consider the moding $p(-), r(+)$ and let $P=\{p(a) \leftarrow\}$ and $Q=p(x), r(x)$. Then $p(a)$ is an SLD-resolvent of $Q$ and $p(a)$ is not simply moded.

However, a simple additional condition does ensure persistence. Namely, we have the following lemma.

Lemma 30 (Simple modedness). Every SLD-resolvent of a simply moded query and a simply moded clause, that is variable-disjoint with it, is simply moded, when the input part of selected atom is an instance of the input part of the head of the clause.

Proof. Omitted.

Corollary 31 (Simple modedness). Let $P$ and $Q$ be simply moded. Consider an $S L D$-derivation $\xi$ of $P \cup\{Q\}$ such that the input part of each selected atom is an instance of the input part of the head of the used clause. Then all queries in $\xi$ are simply moded.

To use this result we now link the delay declarations with matching.

Definition 32. We say that the delay declarations imply matching if for every atom $p(\mathbf{u}, \mathbf{v})$ which satisfies its delay declaration and for every head $p\left(\mathbf{u}^{\prime}, \mathbf{v}^{\prime}\right)$ of a clause if $p(\mathbf{u}, \mathbf{v})$ and $p\left(\mathbf{u}^{\prime}, \mathbf{v}^{\prime}\right)$ unify, then $\mathbf{u}$ is an instance of $\mathbf{u}^{\prime}$.

In particular, if the delay declarations imply the moding, then they imply matching, but not conversely. This brings us to the following conclusion.

Theorem 33 (Correct Typing). Suppose that

- $P$ and $Q$ are well-typed and simply moded,

- the delay declarations imply matching.

Then in all $S L D$-derivations of $P \cup\{Q\}$ which respect the delay declarations every selected atom which is ground in its input positions is correctly typed in its input positions. 
Proof. It is a direct consequence of the Lemmata 27, 29, the Well-typedness Corollary 24 and the Simple modedness Corollary 31.

This result can be used to prove absence of errors in presence of arithmetic relations by using for each arithmetic relation $p$ a delay declaration

$\operatorname{DELAY} P(X, Y)$ UNTIL ground( $X) \wedge \operatorname{ground}(Y)$

and a typing $p(+:$ Gae, $+:$ Gae $)$. In the case of quICKSORT take the typing qs(+ : ListGae, - : ListGae), part(+ : Gae, + : ListGae, - : ListGae, - : ListGae), app(+ : ListGae, + : ListGae, - : ListGae)

$>$ (+: Gae, $+:$ Gae $)$, $\leq(+:$ Gae,$+:$ Gae $)$,

Then QUICKSORT is well-typed (see Apt [Apt93]). Also, it is clearly simply moded. Moreover, the delay declarations from the introduction imply matching. Indeed, if a non-variable term unifies with $\left[\begin{array}{lll}X & \mid & X_{s}\end{array}\right]$, then it is an instance of $\left[\begin{array}{lll}X & \mid & X_{s}\end{array}\right]$.

We conclude that for $s$ a list of integers, all SLD-derivations of QUICKSORT $\cup\{q s(s, x)\}$ which respect the delay declarations from the introduction do not end in an error.

\section{Termination}

Finally, we study termination of logic programs with delay declarations. The key idea in our approach is the restriction to a specific class of SLD-derivations.

\subsection{Termination via Determinacy}

Definition 34. We say that an SLD-derivation is determinate if every selected atom unifies with a variant of at most one clause head, that is, every selected atom can be resolved using at most one clause.

The following simple observation, which is of independent interest, forms the basis of our approach.

Lemma 35. Suppose that

- an $S L D$-derivation of $P \cup\{Q\}$ is successful.

Then all determinate $S L D$-derivations of $P \cup\{Q\}$ are successful, hence finite.

Proof. Consider a determinate SLD-derivation $\xi$ of $P \cup\{Q\}$. $\xi$ is a branch in an SLD-tree $\mathcal{T}$ for $P \cup\{Q\}$. By the strong completeness of the SLD-resolution $\mathcal{T}$ is successful and since $\xi$ is determinate, $\mathcal{T}$ has just one branch, namely $\xi$. So $\xi$ is successful, and a fortiori finite.

To use this result, we link the delay declarations with the notion of determinacy. 
Definition 36. We say that the delay declarations imply determinacy if every atom which satisfies its delay declaration unifies with a variant of at most one clause head.

This brings us to the following result.

Theorem 37 (Termination 1). Suppose that

- an $S L D$-derivation of $P \cup\{Q\}$ is successful,

- the delay declarations imply determinacy.

Then all SLD-derivations of $P \cup\{Q\}$ which respect the delay declarations are successful, hence finite.

Proof. It is an immediate consequence of Lemma 35, because if the delay declarations imply determinacy, then every SLD-derivation which respects these delay declarations is determinate.

Let us see now how to apply this result to the IN_ORDER program. Using the approach of Apt [Apt93] it is straightforward to prove that for a tree $t$, IN_ORDER $\cup\{$ in_order $(t, Y s)\}$ satisfies the first condition of the above theorem. Also, the assumed delay declarations clearly imply determinacy. We conclude that all SLD-derivations of IN_ORDER $U\{$ in_order $(t, Y s)\}$ which respect the delay declarations are finite.

However, the above theorem cannot be directly applied to the QUICKSORT program because the delay declarations from the introduction do not imply determinacy in the case of the part relation. On the other hand, it is possible to adjust these delay declarations and slightly modify the execution of the program so that for the query $q s(s, Y s)$, where $s$ is a list of integers, termination can be established.

Namely, consider the following alternative set of delay declarations for the part relation, given in Naish [Nai88]:

DELAY part (_, [], -, -) UNTIL true.

DELAY $\operatorname{part}(X,[Y \mid],-,-)$ UNTIL ground(X) $\wedge$ ground( $Y)$.

We now say that an atom satisfies a set of delay declarations if it satisfies at least one of them. The idea behind these new delay declarations is to enforce that the arguments of the arithmetic atoms $X>Y$ and $X \leq Y$ are ground once they are introduced through the selection of a part-atom. Note that now the delay declarations for the arithmetic relations become superfluous in the sense that they are always satisfied.

Also, observe that for this new set of delay declarations absence of deadlock is now ensured by the Absence of Deadlock 2 Theorem 26 and not anymore by the Absence of Deadlock 1 Theorem 19. Indeed, these new delay declarations are clearly implied by the typing given in Section 5 but are not any longer implied by the moding given in Section 3, as not all ground terms are of the form [y/ys]. 
Further, note that this new set of delay declarations still implies matching. So the proof of the absence of errors in all SLD-derivations of QUICKSORT $\cup\{q s(s, x)\}$ respecting the delay declarations and given in the previous section remains valid.

However, the determinacy is still not ensured. To deal with this problem we now modify the execution of the programs by viewing the arithmetic atoms as guards in the sense of e.g. Shapiro [Sha89].

\subsection{Termination for Guarded Programs}

By treating atoms as guards we mean the following generalization of the SLDresolution, which we call the SLDG-resolution. By a guarded clause we refer to a construct of the form $H \leftarrow \mathbf{G} \mid \mathbf{B}$, where $H$ is an atom and $\mathbf{G}$ and $\mathbf{B}$ are sequences of atoms. A guarded program is a set of guarded clauses. If $\mathrm{G}$ is empty, then we drop the vertical bar "|". The atoms in $\mathbf{G}$ are called guards. Note that we do not insist that a guarded program is a finite set of clauses. The reason is that we wish to have the possibility of defining the relations used in the guards by a possibly infinite set of ground facts. It is clear how to extend the notions of well-modedness etc. to guarded programs.

We now view QUICKSORT as a guarded program by rewriting the last two clauses defining the part relation as follows:

$\operatorname{part}\left(X,\left[Y \mid X_{8}\right],[Y \mid L s], B 8\right) \leftarrow X>Y \mid \operatorname{part}(X, X 8, L 8, B 8)$. $\operatorname{part}\left(X,\left[Y \mid X_{8}\right], L 8,[Y \mid B 8]\right) \leftarrow X \leq Y \mid \operatorname{part}(X, X 8, L 8, B 8)$.

We call the resulting program QUICKSORT-G. We assume that both in QUICKSORT and in QUICKSORT-G the arithmetic relations are defined by the infinite set of ground facts.

Consider now a query $\mathbf{A}, B, C$ and a variable disjoint with it guarded clause $H \leftarrow \mathbf{G} \mid \mathbf{B}$. We say that $B$ guardedly unifies with $H$ if for some mgu $\theta$ of $B$ and $H$ the query $\mathbf{G} \theta$ is ground and succeeds. We call then $(\mathbf{A}, \mathbf{B}, \mathbf{C}) \theta$ an $S L D G$ resolvent of $\mathbf{A}, B, \mathbf{C}$ and $H \leftarrow \mathbf{G} \mid \mathbf{B}$. So, intuitively, the test that the guards are ground and succeed forms now a part of the unification process. If $\mathbf{G}$ is empty, then the SLDG-resolvents coincide with the SLD-resolvents. So SLDG-resolution is indeed a generalization of the SLD-resolution. It is now clear how to define the SLDG-derivations and the SDLG-trees.

Given a guarded program, we now say that the delay declarations imply determinacy if every atom which satisfies its set of delay declarations guardedly unifies with a variant of at most one guarded clause head.

Note that the new set of delay declarations implies determinacy for the guarded program QUICKSORT-G because in the case of the part relation for any two ground terms $s, t$ at most one of the queries $s>t$ and $s \leq t$ succeeds. The remaining delay declarations imply determinacy because if a non-variable term unifies with $\left[X \mid X_{8}\right]$, then it is does not unify with [ $]$.

Definition 38. We say that a guarded program is regular if 
- every relation used in the guards is defined by a set of ground facts,

- for every other relation symbol $p$ either

- in all clauses defining $p$ all guards are empty, or

- for some sequence of terms $\mathbf{s}$ and a set of variables $V \subseteq \operatorname{Var}(\mathbf{s})$, every guarded clause defining $p$ is of the form

$$
p(\mathbf{s}, \mathbf{t}) \leftarrow \mathbf{G} \mid \mathbf{B}
$$

for some $t, G, B$, such that $\operatorname{Var}(\mathbf{G})=V$.

Note that QUICKSORT-G is regular. In fact, we observed that most of the programs that use arithmetic comparison relations are regular when viewed as guarded programs.

The following theorem explains our approach to the proofs of termination for guarded programs. It is a modification of the Termination 1 Theorem 37.

Theorem 39 (Termination 2). Suppose that

- $P$ is a regular guarded program,

- $P$ and $Q$ are simply moded,

- the delay declarations imply determinacy and matching,

- an SLDG-derivation of $P \cup\{Q\}$ is successful.

Then all $S L D G$-derivations of $P \cup\{Q\}$ which respect the delay declarations are successful, hence finite.

Proof. Omitted.

Let us return now to QUICKSORT-G. In the previous sections we already checked before that QUICKSORT-G with the query qs (s, Ys), where $\mathrm{s}$ is a list of integers, satisfies the first three condtions of the above theorem. Now, using the approach of Apt [Apt93] it is easy to show that QUICKSORT-G $\cup\{q s(s$, Ys $)\}$ satisfies the last condition. We conclude by the Termination 2 Theorem 39 that all SLDG-derivations of QUICKSORT $\cup\{q s(s, Y s)\}$ which respect the modified delay declarations are finite.

It is worthwhile to point out that the SLDG-resolution is very meaningful from the operational point of view. In the case of QUICKSORT-G it prevents a choice of a "wrong" alternative in the definition of the part relation and consequently, obviates a backtracking.

Finally, note that we cannot apply either the Termination 1 Theorem 37 or the Termination 2 Theorem 39 to the SEQUEHCE program, because the delay declaration

DELAY app (_, ,, Z) UHTIL nonvar(Z)

does not imply determinacy. Currently we are working on techniques allowing us to deal with termination in absence of determinacy.

The above two theorems are applicable only to the queries which have successful SLD-derivations. At this moment we know how to deal with termination 
for arbitrary queries at the cost of restricting attention to fair SLD-derivations. Recall that an SLD-derivation is fair if it is finite or if every atom occurring in it is eventually selected (after some possible instantiations).

Below, by an $L D$-derivation we mean an SLD-derivation via the leftmost selection rule. So LD-derivations are SLD-derivations generated by the Prolog selection rule. In the literature a lot of attention has been devoted to the study of termination with respect to the Prolog selection rule (see for example the survey article of De Schreye and Decorte [SD94]). This work can be use to deal with termination in presence of delay declarations. Namely, we have the following result.

Theorem 40 (Termination 3). Suppose that

- all $L D$-derivations of $P \cup\{Q\}$ are finite.

Then all fair $S L D$-derivations of $P \cup\{Q\}$ are finite.

Proof. The proof uses a generalization of the Switching Lemma (see Lloyd[Llo87, pages 50-51] to infinite SLD-derivations. Using it one can prove that if an infinite fair SLD-derivation exists, then an infinite LD-derivation exists. We omit the details.

\subsection{Termination of APPEND}

It is important to realize that in general termination in presence of delay declarations is very subtle. As an example of the difficulties consider the APPEND program augmented with the delay declaration

DELAY $\operatorname{app}(X, \ldots,-)$ UNTIL nonvar $(X)$.

It seems that $\triangle P P E N D$ then terminates for all queries, that is, for all queries $Q$, all SLD-derivations of $\triangle P P E N D \cup\{Q\}$ which respect this delay declaration are finite. The informal argument goes as follows. When the second clause of APPEND can be used to resolve a query of the form $\operatorname{app}(s, t, u)$, then $s$ is of the form $[x \mid x s]$, so in the next resolvent the first argument of app is $x 8$, thus shorter. So eventually, the first argument is either a constant, in which case the second clause cannot be used, or else a variable and in this case the SLD-derivation terminates due to the delay declaration.

However, this reasoning is incorrect. Namely, consider the query app ( [ $X$ । J], Ys, U). Using the second clause it resolves to itself and consequently an infinite SLD-derivation for app $([X \mid J], Y s, \sigma)$ can be generated with the first argument of app always being non-variable. (This fact was noticed in Naish [Nai92] and Lüttringhaus-Kappel [LK93]).

Thus the query app $\left(\left[\begin{array}{lll}X & \mid\end{array}\right], Y_{s}, U\right)$ does not terminate in presence of the delay declaration DELAY app(X, , , ) UNTIL nonvar $(X)$. Similarly, the query $\operatorname{app}(U, Y s,[X \mid \sigma])$ does not terminate in presence of the delay declaration DELAY app (_, -, Z) UNTIL nonvar(Z). 
It seems that the problem has to do with the fact that the first and the third arguments of app share a variable. However, limiting one's attention to the queries of the form $\operatorname{app}(s, t, u)$ where $s, t, u$ are pairwise variable disjoint does not help, as the query $\operatorname{app}\left(\left[\left(\boldsymbol{U}, X_{1}, X 1\right) \mid \sigma\right], Y s,[([\times 2 \mid \nabla 1], W, \nabla 1) \mid W]\right)$ resolves to the above query $\operatorname{app}([X|\bar{X}| \mathrm{J}, \mathrm{Ys}, 0)$.

What does hold is the following limited property.

Theorem 41. Suppose that $\mathrm{s}, \mathrm{t}, \mathrm{u}$ are terms such that $\mathrm{s}$ and $\mathrm{u}$ are variable disjoint.

(i) If $\mathbf{u}$ is linear, then all SLD-derivations of $\mathbb{A P P E N D} \cup\{\operatorname{app}(\mathbf{s}, \mathbf{t}, \mathbf{u})\}$ that respect the delay declaration DELAY app $(\mathrm{X}, \ldots,-)$ UNTIL nonvar $(\mathrm{X})$ terminate.

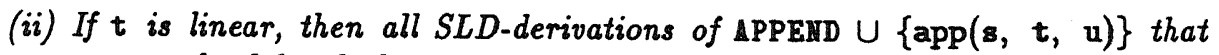
respect the delay declaration DELAY $\operatorname{app}\left({ }_{-}, \ldots, \mathrm{Z}\right)$ UHTIL nonvar(Z) terminate.

(iii) If $\mathbf{s}$ and $\mathbf{t}$ are linear, then all $S L D$-derivations of $\triangle \mathrm{APEND} \cup\{\operatorname{app}(\mathrm{s}, \mathrm{t}, \mathrm{u})\}$ that respect the delay declaration

DELAY $\operatorname{app}(X,-, Z)$ UNTIL nonvar(X) $\vee \operatorname{nonvar}(Z)$

terminate.

Proof. (i) Let $I(v)$ denote the number of symbols of the term v. Suppose that $\operatorname{app}(s, t, u)$ satisfies the delay declaration and resolves to $\operatorname{app}\left(s^{\prime}, t^{\prime}, u^{\prime}\right)$ using the second clause of APPEND. We prove that then $s^{\prime}$ and $u$ ' are variable disjoint, $u^{\prime}$ is linear and the pair $\left(I\left(u^{\prime}\right), I\left(s^{\prime}\right)\right)$ is smaller than $(I(u), I(s))$ in the lexicographic ordering.

8 is not a variable, so it is of the form [x/xs], and $u$ is not a constant. Two cases arise where we assume that $\operatorname{app}(s, t, u)$ and $\operatorname{app}\left(\left[\begin{array}{l|l}X & \mid X_{s}\end{array}\right], Y s,[X \mid\right.$ Zs]) are variable disjoint.

Case $1 \mathrm{u}$ is a variable.

Then $\{x / x, X s / x s, Y s / t, u /[x \mid Z s]\}$ is an mgu of $\operatorname{app}(s, t, u)$ and $\operatorname{app}([X \mid$ $X s], Y s,[X \mid Z s])$, so $\operatorname{app}(s, t, u)$ resolves to $\operatorname{app}(x s, t, Z s)$. Now $x s$ and $\mathrm{Zs}_{\mathrm{s}}$ are variable disjoint, $\mathrm{Zs}$ is linear and the pair $(\mathrm{I}(\mathrm{Zs}), I(x \mathrm{~s}))$ is smaller than $(l(u), l([x \mid x s]))$ in the lexicographic ordering.

Case $2 \mathrm{u}$ is a compound term.

Then $u$ is of the form $[z \mid z s]$. By the Iteration Lemma 1 the substitution $\left\{x / x, X_{s} / x_{8}, Y_{s} / t, Z s / z s\right\} \theta$, where $\theta$ is a relevant $m g u$ of $x$ and $z$, is an mgu of $\operatorname{app}(s, t, u)$ and $\operatorname{app}\left(\left[\begin{array}{l}X \mid X_{s}\end{array}\right], Y s,[X \mid Z s]\right)$. By assumption $x$ is variable disjoint with zs. Moreover, $[z \mid z 8]$ is linear, so $z$ is variable disjoint with $\mathbf{z s}$, as well. Thus by the relevance of $\theta, \mathbf{z s} \theta=\mathbf{z s}$, so $\operatorname{app}(\mathrm{s}, t, u)$ resolves to $\operatorname{app}(x \mathbf{s} \theta, t \theta, z s)$. Now, $z 8$ is linear, so $x \mathrm{~s} \theta$ and $z 8$ are variable disjoint, because $\operatorname{Var}(x \mathbf{s} \theta) \subseteq \operatorname{Var}(\mathbf{s}) \cup \operatorname{Var}(z)$. Moreover, the pair $(1(z \mathrm{z}), I(x \mathrm{~s} \theta))$ is smaller than $(1([z \mid z s]), 1(s))$ in the lexicographic ordering.

This proves the claim.

(ii) By symmetry with (i). 
(iii) Suppose that an infinite SLD-derivation $\xi$ of $\triangle P P E Y D \cup\{\operatorname{app}(\mathbf{s}, t, u)\}$ exists that respects the delay declaration

DELAY $\operatorname{app}(X,-, Z)$ UNTIL nonvar(X) $\vee$ nonvar(Z).

Then either $\mathrm{s}$ or $\mathrm{u}$ is not a variable. Assume without loss of generality that $\mathrm{z}$ is not a variable. By (i) a descendant of $\operatorname{app}(s, t, u)$ in $\xi$ exists which is of the form $\operatorname{app}\left(X_{s}, t^{\prime}, u^{\prime}\right)$ for a variable $X_{s}$ and some terms $t^{\prime}$ and $u^{\prime}$ '. Since $\xi$ is infinite, $u^{\prime}$ ' is not a variable.

In the moding app $(t,+,-)$ \PPEND and the query $\operatorname{app}(s, t, u)$ are nicely moded and the head of every clause of $\triangle$ PPEIVD is input linear. Thus by the Nice modedness Corollary $12 u^{\prime}$ is linear and $X s$ and $u^{\prime}$ are variable disjoint.

By (ii) a descendant of $\operatorname{app}\left(X_{s}, t^{\prime}, u^{\prime}\right)$ in $\xi$ exists which is of the form $\operatorname{app}\left(s^{\prime \prime}, t \prime \prime, Z s\right)$ for some terms $s^{\prime \prime} '$ and $t{ }^{\prime \prime}$ ' and a variable Zs. Moreover, in every descendant of app $\left(X_{s}, t^{\prime}, u^{\prime}\right)$ in $\xi$ the first argument of app remains a variable and consequently $\operatorname{app}\left(s^{\prime} \prime, t, \prime, Z s\right)$ does not satisfy the delay declaration DELAY app(X, , Z) UNTIL nonvar ( $X) \vee$ nonvar(Z). A contradiction.

This isolated result shows how elaborate arguments are sometimes needed to prove simple termination results in presence of delay declarations. In this connection let us mention a related work of Naish [Nai86] and LüttringhausKappel [LK93] who automatically generate delay declarations which ensure that a given program terminates with respect to a selection rule according to which the leftmost non-delayed atom is selected.

\section{Conclusions}

In this paper we dealt with the correctness of logic programs augmented by the delay declarations. To this end we strengthened and adapted methods that were originally developed for the study of Prolog programs.

Interestingly, we can reverse the situation and derive the appropriate delay declarations by analyzing the Prolog programs. Consider for example the QUICKSORT program with the query $q 8(8, Y s)$, where $s$ is a list of integers. Once we have shown in Section 4 that it is well-moded with the moding qs $(+,-)$, partition $(+,+,-,-)$, app $(+,+,-),+>+,+\leq+$ we can augment it by arbitrary delay declarations which are implied by this moding and conclude by virtue of the Absence of Deadlock 1 Theorem 19 that for the query in question no deadlock arises.

Once we have shown in Section 4 that it is well-typed and simply moded with the typing

qs(+ : ListGae, - : ListGae),

part(+ : Gae, + : ListGae, - : ListGae, - : ListGae),

app (+ : ListGae, + : ListGae, - : ListGae)

$>(+:$ Gae,$+:$ Gae $)$,

$\leq(+: G a e,+: G a e)$,

we can augment it by arbitrary delay declarations which imply matching and 
conclude by virtue of the Correct Typing Theorem 33 that for the query in question no run time errors due to the use of arithmetic relations arise.

Finally, once we have shown in Section 6 that some SLDG-derivation of QUICKSORT-G $\cup\{q s(\mathrm{~s}, \mathrm{Is})\}$ is successful, we can augment QUICKSORT-G by arbitrary delay declarations which imply determinacy and matching, and conclude by virtue of the Termination 2 Theorem 39 that all SLDG-derivations of qs ( 8 , Ys) which respect these delay declarations are finite. This shows that it is possible to derive correct parallel logic programs by analyzing Prolog programs.

We conclude by noticing that the "stronger" the delay declarations are the bigger the chance that a deadlock arises, but the smaller the chance that divergence can result. So deadlock freedom and termination seem to form two boundaries within which lie the "correct" delay declarations.

\section{Acknowledgements}

The first author would like to thank Elena Marchiori and Frank Teusink for helpful discussions on the subject of the delay declarations.

\section{References}

[AE93] K. R. Apt and S. Etalle. On the unification free Prolog programs. In A. Borzyszkowski and S. Sokolowski, editors, Proceedings of the Conference on Mathematical Foundations of Computer Science (MFCS 99), Lecture Notes in Computer Science, pages 1-19, Berlin, 1993. Springer-Verlag.

[AP94] K. R. Apt and A. Pellegrini. On the occur-check free Prolog programs. ACM Toplas, 16(3):687-726, 1994.

[Apt90] K. R. Apt. Logic programming. In J. van Leeuwen, editor, Handbook of Theoretical Computer Science, pages 493-574. Elsevier, 1990. Vol. B.

[Apt93] K. R. Apt. Declarative programming in Prolog. In D. Miller, editor, Proc. International Symposium on Logic Programming, pages 11-35. MIT Press, 1993.

[BLR92] F. Bronsard, T.K. Lakshman, and U.S. Reddy. A framework of directionality for proving termination of logic programs. In K. R. Apt, editor, Proceedings of the Joint International Conference and Symposium on Logic Programming, pages 321-335. MIT Press, 1992.

[CC88] H. Coelho and J. C. Cotta. Prolog by Example. Springer-Verlag, Berlin, 1988.

[CP94] R. Chadha and D. A. Plaisted. Correctness of unification without occur check in Prolog. Journal of Logic Programming, 18(2):99-122, 1994.

[DFT91] P. Deransart, G. Ferrand, and M. Téguia. NSTO programs (not subject to occur-check). In V. Saraswat and K. Ueda, editors, Proceedings of the International Logic Symposium, pages 533-547. The MIT Press, 1991.

[DM85] P. Dembinski and J. Maluszynski. AND-parallelism with intelligent backtracking for annotated logic programs. In Proceedings of the International Symposium on Logic Programming, pages 29-38, Boston, 1985.

[Dra87] W. Drabent. Do Logic Programs Resemble Programs in Conventional Languages? In International Symposium on Logic Programming, pages 389-396. San Francisco, IEEE Computer Society, August 1987. 
[HL94] P. M. Hill and J. W. Lloyd. The Gödel Programming Language. The MIT Press, 1994.

[Kow79] R. Kowalski. Algorithm = Logic + Control. Communications of ACM, 22:424-431, 1979.

[LK93] S. Lüttringhaus-Kappel. Control generation for logic programs. In D.S. Warren, editor, Proceedings of the 10th Int. Conf. on Logic Programming, Budapest, pages 478-495. MIT, July 1993.

[Llo87] J. W. Lloyd. Foundations of Logic Programming. Springer-Verlag, Berlin, second edition, 1987.

[Lui94] I. Luitjes. Logic programming and dynamic selection rules. Scriptie (Master's Thesis), University of Amsterdam, 1994.

[MM82] A. Martelli and U. Montanari. An efficient unification algorithm. ACM Transactions on Programming Languages and Systems, 4:258-282, 1982.

[Nai82] L. Naish. An Introduction to MU-PROLOG. Technical Report TR 82/2, Dept. of Computer Science, Univ. of Melbourne, 1982.

[Nai86] L. Naish. Negation and Control in Prolog. Number 238 in Lecture Notes in Computer Science. Springer-Verlag, 1986.

[Nai88] L. Naish. Parallelizing NU-Prolog. In Proceedings of the Fifth Annual Symposium on Logic in Computer Science, pages 1546-1564. The MIT Press, 1988.

[Nai92] L. Naish. Coroutining and the construction of terminating logic programs. Technical Report 92/5, Department of Computer Science, University of Melbourne, 1992.

[SD94] D. De Schreye and S. Decorte. Termination of logic programs: the neverending story. Journal of Logic Programming, 19-20:199-260, 1994.

[Sha89] E. Shapiro. The family of concurrent logic programming languages. $A C M$ Computing Surveys, 21(3):412-510, 1989. 